\title{
EXPERIENCIAS Y REFLEXIONES EN LA ENSEÑANZA DE LA HOMOLOGÍA Y HOMOPLASIA EN EL COLEGIO SECUNDARIO
}

Recibido:21-02-2012

Aceptado: 18-07-2012

\author{
Por: Sergio Urquiza1, Fernando Carezzano², Karina Dorflinger ${ }^{3}$ y Matías Alonso ${ }^{4}$
}

\section{Resumen}

Se discute el rol que la homología y la homoplasia podrían jugar en la enseñanza de la biología evolutiva y de la naturaleza de la ciencia en los últimos años del colegio secundario. La homología es uno de los conceptos más discutidos en Biología y central en biología evolutiva. Permite analizar conceptos de filogenia, biología del desarrollo, genética molecular, morfología y paleontología e integrarlos en diferentes formas, logrando así una visión más acabada e integral de la biología y de los organismos. Se concluye que puede usarse como elemento vertebrador para articular parte de la Biología y abordar a través de él algunos elementos de la naturaleza de la ciencia.

Palabras clave: Biología evolutiva, homología, homoplasia, didáctica de la biología.

\section{Abstract}

We discuss the role that homology and homoplasy might play in the teaching of Evolutionary Biology and the Nature of Science. Homology is one of the most discussed concepts in Biology and it is a main concept in Evolutionary Biology. It allows analyzing concepts of phylogeny, developmental biology, molecular genetics, morphology, and paleontology, and integrates them into different forms, thus achieving more complete and comprehensive view of Biology and organisms. We suggest that Homology be used as backbone to articulate much of Biology and through it to address some elements of the Nature of Science.

Keywords: Evolutionary Biology, homology, homoplasy.

${ }^{1}$ Master en Biología Comparada, profesor asistente y adscripto a la cátedra de Morfología Animal de la Facultad de Ciencias Exactas, Físicas y Naturales de la Universidad Nacional de Córdoba.

2 Profesor asistente en las cátedras de Morfología Animal y Anatomía Comparada de la Facultad de Ciencias Exactas, Físicas y Naturales de la Universidad Nacional de Córdoba.

${ }^{3}$ Estudiante avanzado de la carrera de Ciencias Biológicas de la Facultad de Ciencias Exactas, Físicas y Naturales de la Universidad Nacional de Córdoba.

${ }^{4}$ Estudiante avanzado de la carrera de Ciencias Biológicas de la Facultad de Ciencias Exactas, Físicas y Naturales de la Universidad Nacional de Córdoba. 


\section{Introducción}

La apropiación de los conceptos, métodos y actitudes científicas no es fácil de lograr. En Biología, por ejemplo, suele encontrarse una gran dificultad al operar con las nociones de célula, ser vivo, vida, evolución biológica, selección natural y especie, entre otros. Varias razones explicarían lo anterior. Algunas serían extrínsecas, tales como la calidad de los textos, la capacitación de los docentes o el enfoque educativo imperante (Díaz y García, 2011) mientras otras serían propias del área, como su complejidad y nivel de abstracción (Gándara Gómez et al, 2002; Bar y Valenzuela, 2006). Quizás entre estos factores y otros de índole existencial o religiosa, pueda encontrarse el motivo por el que en adultos educados aún persisten concepciones alternativas a la teoría evolutiva como explicación sobre el origen y dinámica de la vida, así como para diversos problemas científicos, basándose en la creencia de que no existe una verdad objetiva y todo es relativo (Otto, 2011). Si bien es deseable una actitud abierta y pluralista, no es admisible la idea de que toda explicación es aceptable, como si no hubiera posibilidad de resolver tales controversias.

Lo expuesto condujo a la formulación de los siguientes interrogantes acerca de la validación del conocimiento biológico frente a los alumnos: ¿qué enseñar en biología evolutiva? y ¿cómo recrear las maneras en que se abordan los problemas en esta disciplina, reflejando algunos aspectos sobre la naturaleza de la ciencia? Esto llevó a plantearse como objetivos el discutir e implementar la enseñanza de los conceptos de homología y homoplasia en los últimos años del colegio secundario y a analizar la utilización de ambas nociones en el abordaje didáctico de la naturaleza de la ciencia. Dicha discusión se basa tanto en reflexiones teóricas como en experiencias de aula pasadas y otras que se encuentran en desarrollo. Se espera que el abordaje aquí propuesto ayude a los alumnos a diferenciar entre discursos científicos de aquellos que no lo son.

El aprendizaje de las ciencias y particularmente de la biología evolutiva podría ser enriquecido al abordar la diversidad de metodologías de investigación utilizadas, así como la de marcos teóricos y disensos en relación a problemas núcleo. loannidis (2011) plantea que los debates en torno a diferentes hipótesis servirían para discutir la forma o formas en que se hace ciencia permitiendo un mejor aprendizaje de la misma, al inducir la generación de hipótesis y enriquecer la visión del alumnado al sugerir nuevas investigaciones.

Dichas controversias teóricas, reflejan mejor el quehacer científico que el otorgado por la exposición de una visión única y estática, debido a que potencian la productividad científica y la generación de teorías, los productos más importantes del quehacer científico (Adúriz-Bravo e Izquierdo-Aymerich, 2009). Esta aproximación podría representar una forma del saber hacer ciencia, uno de los objetivos de la formación en cultura científica (López Cerezo y Cámara Hurtado, 2010) ya que tal actividad no es solo producción de datos empíricos sino también la generación de hipótesis e interpretación de fenómenos y eventos complejos.

\section{Reflexiones sobre la enseñanza de la homología y homoplasia}

En un análisis sobre la cultura científica como atributo individual, López Cerezo y Cámara Hurtado (2010), plantean que aquella es más que las respuestas correctas a la "ciencia cristalizada" y que debería ir más allá al analizar riesgos, valores, etc. Tal visión 
parece atinada, aunque sin dudas en ciertas ocasiones es necesario implementar un enfoque restringido y reduccionista para poder describir un objeto, evento o fenómeno. Brigandt (2011) afirma que la filosofía de la ciencia en general, y en particular la referida a la biología experimental, habría incorporado últimamente en su análisis el contexto de descubrimiento. Esto implica considerar los condicionamientos sociales, económicos, etc. que rodean al trabajo científico, al igual que los intereses, creencias y valores sustentados por los científicos. El abordaje histórico de la formación de un concepto clarifica su significado al poner de manifiesto los factores mencionados, y es una aproximación muy eficaz para enseñar numerosas temáticas biológicas (Kleisner, 2007). El par de nociones analizadas son muy particulares de la Biología, cuya filosofía deja de lado cuestiones generales, como la discusión de modelos y leyes y se centra en sus propios problemas (Pradeu, 2009) de los que aquellos son centrales.

Osorio y Martins (2010) afirman que no existe acuerdo en las orientaciones, finalidades y enfoques en la enseñanza de las ciencias, por esto deben buscarse metodologías propias. Entonces, ¿cómo proceder en la enseñanza de los conceptos, métodos y actitudes de la naturaleza de la ciencia, y particularmente de la Biología? Quizás una respuesta posible a esto se encuentre en el trabajo de Adúriz-Bravo y Izquierdo-Aymerich (2009), basado en parte en las ideas de Giere, respecto a que un modelo no necesariamente debe ser una propuesta formalizada, sino que puede ser una imagen, una maqueta, etc., siempre y cuando permita reflexionar sobre los fenómenos abordados. Entonces es posible afirmar que debe estimularse al alumnado a realizar sus propios modelos explicativos del mundo y, en el caso que nos ocupa, de la evolución biológica. Estas nociones podrían servir para orientar en la enseñanza de conceptos tan abstractos pero sumamente útiles y vertebradores como los de homología, homoplasia e innovación fenotípica, diferentes estadios de un continuum en la evolución de los organismos. También podrían tenerse en cuenta para el abordaje de las metodologías de estudio que se utilizan en estos trabajos.

Por lo dicho, se considera que proponer al alumno una serie de actividades que ilustren sobre el contexto de descubrimiento de la homología-homoplasia, así como sobre la metodología utilizada en su estudio, serían no solo pertinentes sino esenciales para abordar la naturaleza del conocimiento de la Biología.

\section{La homología, homoplasia y el origen de las innovaciones evolutivas ¿en el centro de la discusión?}

La explicación de casi todo fenómeno biológico se enfrenta al problema de una enorme complejidad, de ahí que frecuentemente se simplifica la explicación, sacrificando por ejemplo el abordaje de la metodología científica. La morfología evolutiva se presta como pocas áreas para integrar diversas disciplinas, conceptos y metodologías. Quizás sería pertinente discutir su implementación en los últimos años del secundario, cuyos alumnos presentan un mayor nivel de abstracción, ya que, el actual, es un momento histórico en que se unen la anatomía, morfología, paleontología, genética y biología del desarrollo entre otras, para intentar dar cuenta del origen y evolución del cuerpo animal (Urquiza et al, 2012a; Urquiza y Carranza, 2012).

La homología es uno de los conceptos más importantes y discutidos en Biología, y además de poseer relevancia práctica, en diversos campos ha estimulado multitud de reflexiones teóricas (Brigandt y Griffiths, 2007; Kleisner, 2007; Pavlinov, 2011). Este concepto fue formado a la luz de debates en épocas anteriores al evolucionismo, y luego se transformó quizás en el principal problema de este último, junto al de la especiación. Richard Owen, a mediados del siglo XIX, definió a la homología como "el mismo órgano en diferentes animales con diferente forma y función". Él ya había identificado un "orden" 
o "patrón común" en los seres vivos aún antes de la publicación del libro sobre el origen de las especies de Darwin. Y, luego de la edición de dicho trabajo, comenzó un debate sobre este término que aún no termina. Aunque para fines del siglo XIX y principios del XX ya tenía un significado más o menos similar al actual (Kleisner, 2007), se han multiplicado las definiciones y los alcances del concepto según la disciplina y uso que se les da, al punto que en la actualidad se han publicado numerosas monografías específicas sobre el tema (Sanderson y Hufford, 1996; Bock y Cardew, 2007; Brigandt y Griffiths, 2007; Pavlinov, 2011).

La homología siempre fue contrapuesta a la analogía, y últimamente a la homoplasia. Si bien en los manuales frecuentemente aparecen enfrentadas al estilo blanco-negro, Lamarck-Darwin, la verdad es que en muchos casos sería mejor hablar de homología parcial (Hall, 2007; Thanukos, 2008; Brigandt, 2011) debido a la ocurrencia de la homocracia u homología profunda (Wake et al, 2011). Esto es, en diferentes organismos alejados filogenéticamente hay órganos o sistemas de estos que poseen genes o vías de desarrollo comunes. Sin embargo, dichos órganos no estaban presentes en el último ancestro común a los linajes en cuestión, por lo tanto, no serían homólogos. Esto podría aplicarse a los ojos complejos o a las extremidades de artrópodos y vertebrados.

La importancia de esta problemática en Biología se debe a que para explicar el patrón y el proceso evolutivos debe establecerse correctamente la homología, convergencia o paralelismo de un carácter (Scotland, 2011). Esto porque al estudiarse la historia de un grupo y sus cambios filogenéticos, morfológicos y de hábitat podrán comprenderse los factores ecológicos y ambientales que llevaron a tales modificaciones. Además ayuda a predecir qué carácter podría encontrarse entre diferentes taxones al poder comparar sus relaciones filogenéticas y sus adaptaciones (Petto y Mead, 2009). Es un tema central porque ilustra claramente la evolución de los órganos y del cuerpo de los animales, pudiendo transformarse en una evidencia de un gran peso en la discusión evolucionismo-antievolucionismo.

La homoplasia es una similitud entre diferentes organismos que no heredaron dicha semejanza de un ancestro común y normalmente es reconocida por incongruencias en el patrón de distribución de caracteres (Thanukos, 2008). Puede ser de tres tipos: paralelismo, convergencia y reversión, siendo las dos primeras mucho más frecuentes. El paralelismo es la producción de estructuras similares desde programas de desarrollo equivalentes y se da en organismos emparentados, o sea que la característica, si bien evolucionó por carriles diferentes en los distintos linajes, llega a un estado final más o menos similar en estos, gracias tanto a presiones evolutivas como a un bagaje genético y de desarrollo parecidos. Por tanto el paralelismo puede ser muy difícil de diferenciar de una homología. La convergencia se debe a la ocurrencia de estructuras similares generadas a partir de programas de desarrollo diferentes, por ejemplo, la típica forma hidrodinámica de peces y ballenas. La reversión se produce cuando aparece una estructura en un organismo que no ha estado presente en las especies anteriores intermedias pero sí en alguna basal más o menos alejada. Se considera que la base genética sería la misma o muy similar a la especie ancestral, pero no es vista como una homología porque el carácter no fue heredado de un ancestro común a otros. Se supone que estos fenómenos se dan porque existen pocas respuestas para adaptarse a ciertas presiones evolutivas. O sea, no puede responderse de cualquier manera debido a la presencia de restricciones. También puede deberse a que se comparten antiguas vías de desarrollo (Wake et al, 2011) o ambos factores a la vez.

Un ejemplo de homoplasia sería el gen pax 6, que dirige la formación de los ojos, el que está presente en casi todos los animales. Probablemente fue heredado desde el ancestro común a estos, pero fue cooptado en diferentes sistemas ópticos en forma 
independiente. Además numerosas tejidos o células serían homólogos en este sistema en distintos animales, aunque los ojos complejos en sí habrían evolucionado en forma independiente (Thanukos, 2008).

También el sistema nervioso es un interesante ejemplo evolutivo. Todos los sistemas nerviosos comparten numerosos genes que dirigen su formación así como la del cuerpo. Sin embargo, no todos los autores coinciden en que estos genes habrían participado de la misma manera en el último ancestro común de los animales bilaterales, el urbilateria. Esto significa que hay quienes consideran que este animal no poseía un sistema nervioso avanzado ni centralizado, y que esto se logró en forma independiente en diferentes linajes (Moroz, 2009). Otra posición sería la que considera que todos los sistemas nerviosos son homólogos, ya que el urbilateria habría poseído uno centralizado y relativamente avanzado, del que derivaron todos los sistemas nerviosos de los linajes animales (Arendt et al, 2008).

Debe remarcarse que el paralelismo y la convergencia están sometidos a una gran discusión desde hace un buen tiempo, y sus significados son a veces contradictorios y sus límites están muchas veces mal definidos. Tan es así que puede ocurrir que fenómenos similares son adscritos a una u otra categoría (Scotland, 2011).

Como se ve, estudiar las homologías no es fácil. Con el advenimiento de la biología molecular parecía que todos los problemas de inferencia filogenética y de determinación de homologías podrían solucionarse, y de hecho, en muchos casos ayuda a clarificar y distinguir entre homologías y homoplasias (Wake et al, 2011), pero pronto se vio que también en este nivel se producen paralelismos, convergencias y demás problemas, al punto de que a pesar de ingentes esfuerzos en todos estos años y de disecarse a nivel molecular numerosos sistemas orgánicos, aún no se llega a un acuerdo sobre su homología o no. Además pueden producirse los mismos tipos celulares y tejidos, por la convergencia en programas de desarrollo (que utilizan diferentes vías genéticas), lo que significa que no son homólogos, sino homoplásicos. Pero esto no es todo, el comportamiento y sobrevida de tejidos similares provenientes de distintos orígenes embrionarios en ciertos trasplantes es muy diferente, lo que lleva a considerar la homoplasia y homología ya no solo por su importancia teórica (por ej. al reflexionar sobre la evolución) o práctica (al determinar filogenias), restringidas a la biología básica, sino por sus implicancias clínicas para la prolongación y mejoramiento de la vida o tratamiento de enfermedades degenerativas (Graham, 2010).

\section{Implicancias para la enseñanza}

Por experiencia y por teoría se sabe que no hay una sola manera de enseñar ni de aprender, por lo que el docente siempre debe estar atento a modificar los cursos de acción, pero fundamentando estos cambios sobre principios epistemológicos, disciplinares y didácticos. Teniendo esto en mente podrán discutirse algunas ideas básicas sobre la temática de la homología.

El hecho de que sea un tema activamente discutido permite abordar problemas de verdadera actualidad, en muchos casos con implicancias muy serias (Graham, 2010; Davies y Lineweaver, 2011) que involucran incluso a la salud humana, con todas las aristas socioeconómicas de imaginar y posibles de analizar en ese contexto. Estas controversias brindan un panorama de cómo se trabaja en ciencia, exhibiendo cómo nacen y se contrastan las hipótesis. Muchas de estas podrán ser analizadas por alumnos de los últimos años de la escuela secundaria, dado que existe esta discusión en todos los niveles de complejidad biológicos, lo que otorga la posibilidad de un diálogo entre varios grupos etarios. Sin embargo, la problemática que pueda girar en torno al tema, debe ser 
cuidadosamente seleccionada, porque fácilmente puede derivar hacia otros carriles, por ejemplo, los problemas médicos, una tendencia común y razonable entre el alumnado.

La discusión en torno a si el par homología-homoplasia es mutuamente excluyente (extremos) o admite gradaciones, al ser parte de un continuum (Hall, 2007), es muy importante como un ejemplo de la labor científica, ya que en Biología, en la ciencia y en la vida cotidiana siempre suelen darse estas discusiones, polarizándose las posiciones, muchas veces por una interpretación errónea de los hechos. Tal intercambio de ideas podría ilustrar sobre que siempre es necesario recabar más información desde otros niveles para encontrar una respuesta.

En la escuela secundaria podría tratarse esta temática mediante la evolución de los fotorreceptores y ojos. Como se vio, la homología parcial y homología profunda podría explicar la evolución de este y otros sistemas de órganos. Podrá analizarse el patrón evolutivo de la presencia de estas estructuras y la homología y homoplasia que se dan a diferentes niveles. Aquí pueden verse varios niveles de homología (molecular, de tejidos, de órganos, etc.), por lo que podrá seleccionarse de acuerdo a la edad del alumnado.

Otros temas posibles son la evolución del sistema nervioso y la de las extremidades. Ambas estructuras están presentes en casi todos los animales, o al menos, en diferentes taxones pertenecientes a los principales grupos. Esto permitiría, por ejemplo, discutir y comparar en anélidos, artrópodos y vertebrados, algunas propuestas muy interesantes como la que postula que el palio de los vertebrados sería homólogo al cuerpo pedunculado de los anélidos, algo impensado hasta hace pocos años.

Entre las metodologías utilizadas en estos estudios que podrían ser abordadas en el aula se cuentan la comparación anatómica y embriológica, la expresión génica diferencial y el análisis del patrón del desarrollo (Urquiza y Carezzano, 2011; Urquiza y Carranza, 2012; Urquiza et al, 2012a). Claro está que con un cuidado muy especial en la forma en la que se lo hace, dado el nivel de abstracción que requieren algunas de aquellas para ser comprendidas. En las experiencias referidas aquí se hizo mucho hincapié en la interpretación de árboles evolutivos, imágenes de patrones de expresión génica y cómo se obtenían estas.

Si bien numerosas hipótesis de homología morfológica son muy discutidas, tienen un gran sustento experimental y teórico, lo que enriquece la visión del alumno. El resultado final, si son o no homólogos, casi carece de importancia cuando se enfrenta a la clase. Lo importante es lograr una conexión del alumno con el tema. Y mediante este enfoque, según experiencias propias, se logran recrear ciertas discusiones muy interesantes entre los alumnos, en las que surgen numerosos interrogantes a través de la realización de diversas actividades.

\section{Experiencias de aula en la enseñanza de la homología y la homoplasia}

Las actividades se desarrollaron los tres últimos años $\left(4^{\circ}, 5^{\circ}\right.$ y $\left.6^{\circ}\right)$ de dos instituciones educativas de nivel medio de la República Argentina. Las mismas consistieron en la lectura, análisis y discusión de diversos textos redactados ad hoc, los que tratan acerca de la evolución del cráneo, del sistema nervioso central, o de la evolución animal en general (Urquiza et al, 2012b; Urquiza y Carranza, 2011). Luego los alumnos respondieron una serie de cuestionarios semiestructurados, a la vez que realizaron una exhaustiva búsqueda de información orientada por el docente (textos, imágenes e ilustraciones de los animales y sistemas de órganos en discusión, árboles filogenéticos, etc.). Se contextualizó esta búsqueda en el momento histórico correspondiente, como por ejemplo, que el concepto de homología, a pesar de ser una de las evidencias más fuertes de la ocurrencia de la evolución, se originó fuera de esta teoría y tenía otra significación para aquellos autores. Estas actividades propiciaron, por 
ejemplo, que algunos alumnos de un quinto año generasen un grupo de discusión en la web, en la cual explicaban y ayudaban a otros preparándolos para las evaluaciones. También se plantearon interesantes discusiones, por ejemplo, con los típicos interrogantes acerca de si todo fue creado por Dios o no, o si los "monos" son los antepasados del hombre. Se logró operar con el concepto de gen y fenotipo, de homología profunda, y efectuar comparaciones pertinentes de homologías y homoplasias en los distintos niveles de complejidad. Lograron operar asimismo con árboles evolutivos simplificados del reino animal, aplicando en ellos el concepto de homología profunda con algunos órganos.

Entre los procesos generados se dieron la comparación entre estructuras morfológicas, inferencias filogenéticas, interpretación de árboles evolutivos e imágenes de patrones de desarrollo, etc. Es de destacar, sin embargo, que aunque el rendimiento no fue homogéneo, se alcanzaron en algunos casos muy buenos resultados.

\section{Conclusiones}

El hecho de estar ante un proceso incipiente de integración conceptual para explicar la evolución fenotípica, puede brindar herramientas e instrumentos extra a la hora de explicar y abordar el problema del origen y la evolución de los organismos. Para esto el análisis y discusión de las homologías y homoplasias de diferentes niveles es crucial, a la vez que ilustra en forma clara y contundente el proceso evolutivo.

Seguir el patrón filogenético de distintos órganos u otras características, además de ser en sí mismo un objetivo, sirve como disparador y medio de reflexión. Permite discutir acerca de embriología, genética molecular, patrones evolutivos, filogenia, etc. Le otorga a la morfología un sentido de coherencia. Debe brindarse la posibilidad de discutir el contexto histórico de los autores y las implicancias de sus creencias: esto es muy atractivo para el alumnado y despierta su curiosidad.

Además, brindar una visión más completa y crítica del patrón y del proceso evolutivo, genera en los estudiantes una visión menos antropocéntrica sobre el mundo y los seres vivos, al percatarse de las similitudes de origen con otros organismos. Esto, es esperable, redundará en un mayor respeto por el medio ambiente y por la vida.

Este es un momento excitante y estimulante por los descubrimientos y hallazgos que se vislumbran en un futuro no muy lejano y el desafío es, entonces, abordar en la escuela temas centrales y articuladores que permitan acceder a este rico e inabarcable universo del conocimiento. 


\section{Referencias}

ADURIZ-BRAVO, A. \& IZQUIERDO-AYMERICH, M. (2009). Un modelo de modelo científico para la enseñanza de las ciencias naturales. Revista Electrónica de Investigación en Educación en Ciencias 4(1): 40-49.

ARENDT, D., DENES, A.S., JEKELY, G., \& TESSMAR-RAIBLE, K. (2008). The evolution of nervous system centralization. Philos. Trans. R. Soc. Lond. B Biol. Sci. 363, 15231528.

BAR, A.I R. \& VALENZUELA, S. D. (2006). La evolución como modelo de explicación en los textos escolares. Red de Revistas Científicas de América Latina y el Caribe, España y Portugal. http://redalyc.uaemex.mx/pdf/101/10102602.pdf [Consulta nov. 2011].

BRIGANDT, I. (2011). The dynamics of scientific concepts: The relevance of epistemic aims and values. En: FEEST, U. \& STEINLE, F. (Eds.). Scientific concepts and investigative practice.

BRIGANDT, I. \& GRIFFITHS, P. (2007). The importance of homology for biology and philosophy. Biology and Philosophy 22: 633-641.

BOCK G \& G. CARDEW (eds.) (2007). Novartis Foundation Symposium 222 - Homology John Wiley \& Sons, Ltd., Chichester, UK

DAVIES, P. C. W. \& LINEWEAVER, C. H. (2011). Cancer tumors as Metazoa 1.0: tapping genes of ancient ancestors. Phys. Biol. 8015001

DÍAZ, I. \& GARCÍA, M. (2011). Más allá del paradigma de la alfabetización. La adquisición de cultura científica como reto educativo. Formación universitaria 4(2): 3-14.

GÁNDARA GÓMEZ, M.; GIL QUÍLEZ, M. J. \& SANMARTÍ PUIG, N. (2002). Del modelo científico de adaptación biológica" en los libros de texto de enseñanza secundaria obligatoria. Enseñanza de las Ciencias 20(2): 303-314.

GRAHAM, A. (2010). Developmental homoplasy: convergence in cellular differentiation. Journal of Anatomy 216: 651-655.

HALL, B. K. (2007). Homoplasy and homology: Dichotomy or continuum? Journal of Human

Evolution 52: 473-479.

IOANNIDIS, S. (2011). Regulatory evolution and theoretical arguments in Evolutionary Biology. Science \& Education (27 jul): 1-14.

KLEISNER, K. (2007). The formation of the theory of homology in biological sciences. Acta Biotheoretica 55: 317-340. 
LÓPEZ CEREZO, J. A. \& CÁMARA HURTADO, M. (2010). Percepción, cultura científica y participación en Iberoamérica. En: Ciencia, tecnología y universidad en Iberoamérica. Coord.: ALBORNOZ, M. \& LÓPEZ CEREZO, J. A. Editorial Eudeba, Buenos Aires.

MOROZ, L. (2009). On the origins of complex brains and neurons. Brain, Behavior and Evolution 74: 177-190.

OSORIO, C. \& MARTINS, I. P. (2010). La educación científica y tecnológica para el Espacio Iberoamericano de Conocimiento En: Ciencia, tecnología y universidad en Iberoamérica. Coord.: ALBORNOZ, M. \& LÓPEZ CEREZO, J. A. Editorial Eudeba, Buenos Aires.

OTTO, S. (2011). Unscientific America: Decline and Fall. The New Scientist 29 (212): 2836.

PAVLINOV, I. (2011). The contemporary concepts of homology in biology: A theoretical review. Biology Bulletin Reviews 2(1): 36-54.

PETTO, A. J. \& MEAD, L. S. (2009). Homology: Why we know a whale is not a fish. Evolution Education and Outreach 2: 617-621.

PRADEU, T. (2011). Philosophie de la biologie. En: BARBEROUSSE, A. \& COZIC, M. Précis de philosophie des sciences. Paris, Vuibert.

SANDERSON, M \& HUFFORD L. (Eds.) (1996). Homoplasy: The Recurrence of Similarity in Evolution. Editorial Academic Press, San Diego, California.

SCOTLAND, R. W. (2011). What is parallelism? Evolution \& Development 13(2): 214-227.

THANUKOS, A. (2008). Bringing homologies into focus. Evolution Education and Outreach $1: 498-504$.

URQUIZA, S. \& CAREZZANO, F. (2011, octubre). ¿Es posible enseñar Morfología Animal evolutiva? Poster presentado en III Congreso Nacional de Anatomistas; II Congreso Internacional de Educación e Investigación en Ciencias Morfológicas; I Jornadas Interdisciplinarias de Morfología y Patología Estructural y Molecular. Córdoba, Argentina.

URQUIZA, S.; CAREZZANO, F.; DORFLINGER, K.; ALONSO, M.; IBARRA, C. \& LUDUEÑA, F. (2012a, mayo). ¿Cómo contribuir desde la biología evolutiva a la construcción de una cultura científica? Poster presentado en XI Jornadas de Ciencias Naturales del Litoral y III Reunión Argentina de Ciencias Naturales. Córdoba, Argentina.

URQUIZA, S.; CAREZZANO, F. y LUDUEÑA, F. (2012b). Relaciones evolutivas del sistema nervioso. Manuscrito no publicado. Cátedra de Morfología Animal, Escuela de Biología, Universidad Nacional de Córdoba. Córdoba, Argentina.

URQUIZA, S. \& CARRANZA, M. (2011). La transición del sistema nervioso entre invertebrados y Vertebrados. Manuscrito no publicado. Cátedra de Morfología 
Animal, Escuela de Biología, Universidad Nacional de Córdoba. Córdoba, Argentina.

URQUIZA, S. \& CARRANZA, M. (2012, mayo). La integración conceptual en Morfología Evolutiva como objetivo educativo. Poster presentado en XI Jornadas de Ciencias Naturales del Litoral y III Reunión Argentina de Ciencias Naturales. Córdoba, Argentina.

WAKE, D. B.; WAKE, M. H. \& SPECHT, C. D. (2011). Homoplasy: from detecting pattern to determining process and mechanism of evolution. Science 331:1032-1035. 